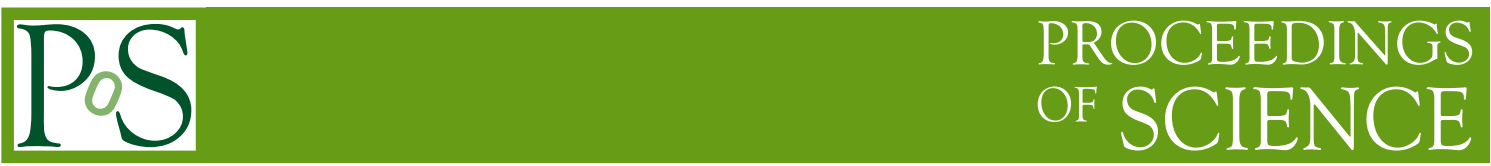

\title{
Single Top Measurement Highlights at CMS
}

\author{
Mintu Kumar ${ }^{a, *}$ \\ ${ }^{a}$ Tata Institute of Fundamental Research, \\ Homi Bhabha Road, Mumbai, India \\ E-mail: mintu.kumar@cern.ch
}

The $t$-channel and $\mathrm{tW}$-channel are the two most dominant single top quark production processes at the LHC. These processes provide a unique phase space at lower interaction scale with lower colour reconnection probability compared to the top quark pair production. The $t$-channel (tWchannel) final state comprises a single top quark produced along with a light quark (W boson) leading to at least two (three) jets, one of which arises from the hadronization of a b-quark, an isolated energetic lepton (electron or muon), and large missing momentum due to a neutrino escaping from the $\mathrm{W}$ boson decay. In this report, measurements of the top quark mass in single top $t$-channel process and of the production cross-section in tW-channel are reported based on $35.9 \mathrm{fb}^{-1}$ of proton-proton (pp) collision data recorded at $13 \mathrm{TeV}$ by the CMS experiment during 2016. A multivariate technique relying on boosted decision trees is deployed to optimally separate signal from background in both measurements. A simultaneous maximum-likelihood fit has been performed in electron and muon final states. The response of the boosted decision trees has been used in a binned likelihood fit to extract the tW-channel cross-section. The measured signal strength and cross section are $1.24 \pm 0.18$ and $89 \pm 4$ (stat) \pm 12 (syst) pb, respectively. The top quark mass is obtained by fitting its reconstructed mass distribution using a suitable combination of parametric shapes to model the $t$-channel signal and various backgrounds. The measured mass of the top quark is found to be $172.13_{-0.77}^{+0.76} \mathrm{GeV}$, reaching a sub-GeV precision for the first time in this specific phase space. The top quark to top antiquark mass ratio and difference are also calculated using the separately measured top and top antiquark mass depending on the lepton charge in the final state.The calculated mass ratio and difference are $0.9952_{-0.0104}^{+0.0079}$ and $0.83_{-1.35}^{+1.79}$ $\mathrm{GeV}$.

The Ninth Annual Conference on Large Hadron Collider Physics - LHCP2021

7-12 June 2021

Online

${ }^{*}$ Speaker

(c) Copyright owned by the author(s) under the terms of the Creative Commons 


\section{Introduction}

A precise measurement of the top quark mass is of profound importance, being an important parameter of the standard model (SM). It constitutes a major input to verify the self-consistency of the SM. Among all elementary particles it is the largest contributor in terms of radiative corrections to the mass of the Higgs boson. The cross-section measurement is also very important to validate the BSM searches since the single top processes are sensitive to the Cabibbo-Kobayashi-Maskawa matrix element $V_{\mathrm{tb}}$, and their study provides a direct probe of this value. The ratio of the top quark to antiquark cross-section i.e. $R_{t}=\sigma(\mathrm{t}) / \sigma(\overline{\mathrm{t}})$ is sensitive to parton distribution function, which helps to study the valance $\mathrm{u}$ to $\mathrm{d}$ quark density. The $t$-channel and $\mathrm{tW}$-channel have total cross section of $216.99_{-7.71}^{+9.04} \mathrm{pb}$ and $71.7 \pm 3.8 \mathrm{pb}$, respectively, calculated at next-to-leading order.

\section{Event reconstruction and categorization}

In both analyses candidate events are selected by requiring at least one isolated lepton and a large missing momentum due to the escaping neutrino. Exactly two (three or more) jets are required in the $t$-channel (tW-channel), out of which one must be a b-tagged jet.

The event categories are defined according to the number of total jets and b-tagged jets $(n \mathrm{~J} m \mathrm{~T})$. The $2 \mathrm{~J} 1 \mathrm{~T}$ category is the largest contributor to $t$-channel signal events and used for the measurement of the top quark mass. The four-momentum of the top quark (and hence its mass) is reconstructed from that of its decay products: the charged lepton, the neutrino, and the b-tagged jet.

Three categories $2 \mathrm{~J} 1 \mathrm{~T}, 3 \mathrm{~J} 1 \mathrm{~T}$ and $4 \mathrm{~J} 1 \mathrm{~T}$ are used for the measurement of the production cross section in the $\mathrm{tW}$-channel. The $3 \mathrm{~J} 1 \mathrm{~T}$ category is the largest contributor to $\mathrm{tW}$-channel signal events and the other two categories are used to constrain the background contribution.

\section{Estimation of the QCD multijet background}

Since QCD multijet production has a small acceptance in the phase space used in these analyses, QCD templates are derived from sideband data for each event category separately. For the mass measurement in the $t$-channel process [2], the QCD contribution in the signal region is estimated by means of a two-component binned maximum-likelihood fit to the distribution of transverse mass $\left(m_{\mathrm{T}}\right)$ of the charged lepton and neutrino system, as shown in Fig. 1 with QCD and nonQCD events being treated as the two fit components. To reject the $\mathrm{QCD}$ contribution, $m_{\mathrm{T}}>50 \mathrm{GeV}$ is required for the further analysis. This method is validated in the QCD dominated category 2J0T. For the cross-section measurement in the tW-channel [1], the QCD contribution in the signal region is estimated by means of a three-component binned maximum-likelihood fit to the $m_{\mathrm{T}}$ distribution with QCD, top quark, and $\mathrm{V}+$ jets events being treated as the three fit components.

\section{Multivariate analysis (MVA)}

A number of kinematic variables are combined into an MVA discriminator to optimally separate single top quark events from backgrounds. These variables are selected such that they have a significant power to distinguish signal and backgrounds. For the top quark mass analysis, they 

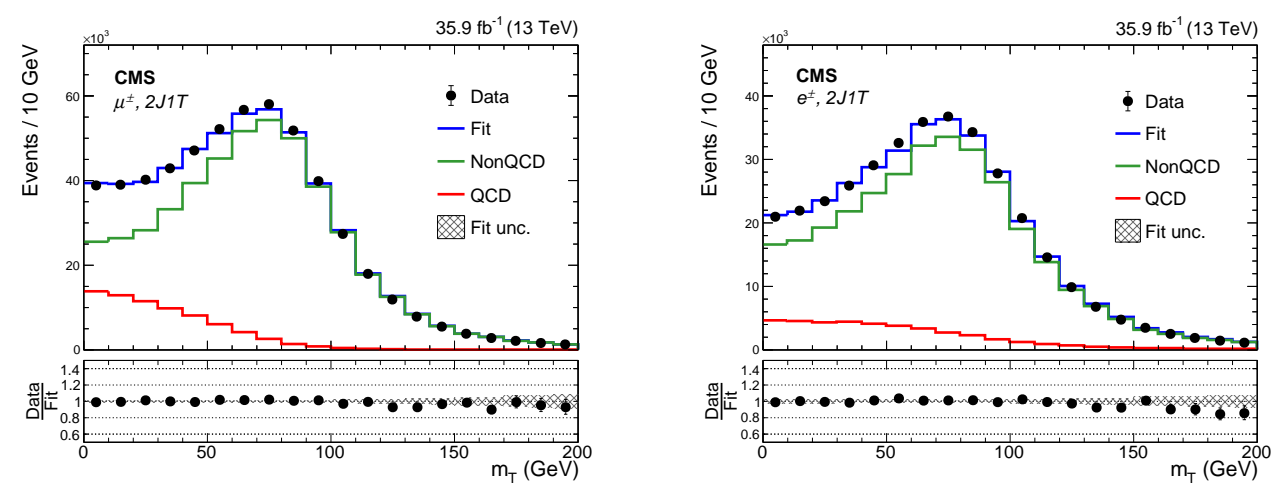

Figure 1: Postfit distributions of $m_{\mathrm{T}}$ for the muon (left [2]) and electron (right [2]) final state.

also need to have a low correction with the reconstructed top quark mass. A criterion on the BDT response $>0.8$ has been applied (Fig. 2), which results in 64\% (58\%) signal purity and $20 \%$ efficiency for the muon (electron) final state. For the cross-section measurement, a BDT training has been done in the $3 \mathrm{~J} 1 \mathrm{~T}$ region while the resulting weights are applied to all categories. The full BDT response has been used as the final observable in the measurement.

\section{Top quark mass measurement overview}

The distributions $y=\ln m_{\mathrm{t}}$ obtained from the muon and electron final states are considered in a simultaneous maximum-likelihood fit. The QCD multijet contribution is subtracted from data before the fit and the remaining distribution is described by a parametric model as:

$$
\mathrm{F}\left(y ; y_{0}, \mathrm{f}_{\mathrm{t}-\mathrm{ch}}, \mathrm{f}_{\text {Top }}, \mathrm{f}_{\mathrm{EWK}}\right)=\mathrm{f}_{\mathrm{t}-\mathrm{ch}} \cdot \mathrm{F}_{\mathrm{t}-\mathrm{ch}}\left(y ; y_{0}\right)+\mathrm{f}_{\mathrm{Top}} \cdot \mathrm{F}_{\mathrm{Top}}\left(y ; y_{0}\right)+\mathrm{f}_{\mathrm{EWK}} \cdot \mathrm{F}_{\mathrm{EWK}}(y)
$$

Here $\mathrm{F}_{\mathrm{t}-\mathrm{ch}}, \mathrm{F}_{\mathrm{Top}}$ and $\mathrm{F}_{\mathrm{EWK}}$ are described by a sum of an asymmetric Gaussian core with a Landau tail, a Crystal ball function [8], a Novosibirsk function [4] to model the signal, top background, and electroweak background, respectively. The normalization scale factors $\mathrm{f}_{t-\mathrm{ch}}, \mathrm{f}_{\text {Top }}$, and $\mathrm{f}_{\text {EWK }}$ are constrained using log-normal priors with $15 \%, 6 \%$, and $10 \%$ based on their respective cross-section results [3] [5-7]. These constraints are included as nuisance parameters in the final fit whereas other systematic sources are externalised. The top quark mass is obtained from the postfit $\ln \left(m_{\mathrm{t}} / 1 \mathrm{GeV}\right)$ distribution, as shown in Fig. 2, by taking the exponential of the postfit value of parameter of interest $y_{0}$.

\section{Cross-section measurement strategy}

A simultaneous binned likelihood fit assuming Poisson distributions is performed in all categories and in both lepton channels on the BDT discriminants in order to extract the tW signal strength. The signal strength parameter is defined as $\mu=\sigma / \sigma_{\text {SM }}$ where $\sigma$ is the measured signal cross section and $\sigma_{\mathrm{SM}}$ is the SM prediction of the cross section. In this analysis systematic uncertainties are included as nuisance parameters in the fit. Fig. 3 shows the BDT discriminant for the signal and control regions scaled to the output of the fit. 

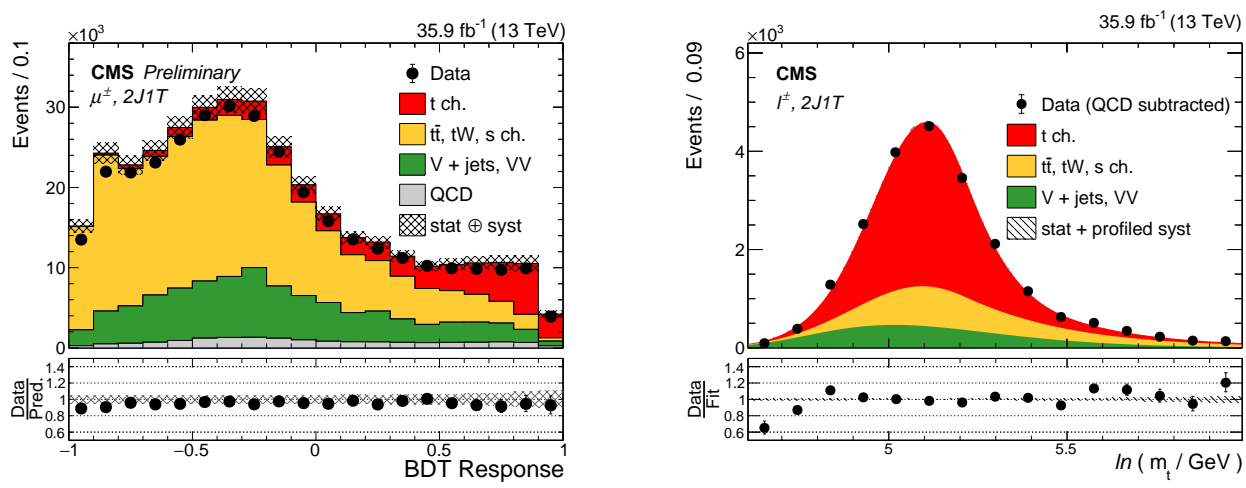

Figure 2: Prefit distribution of the BDT response (left [2]) and postfit distribution of $\ln m_{\mathrm{t}}$ (right [2]).
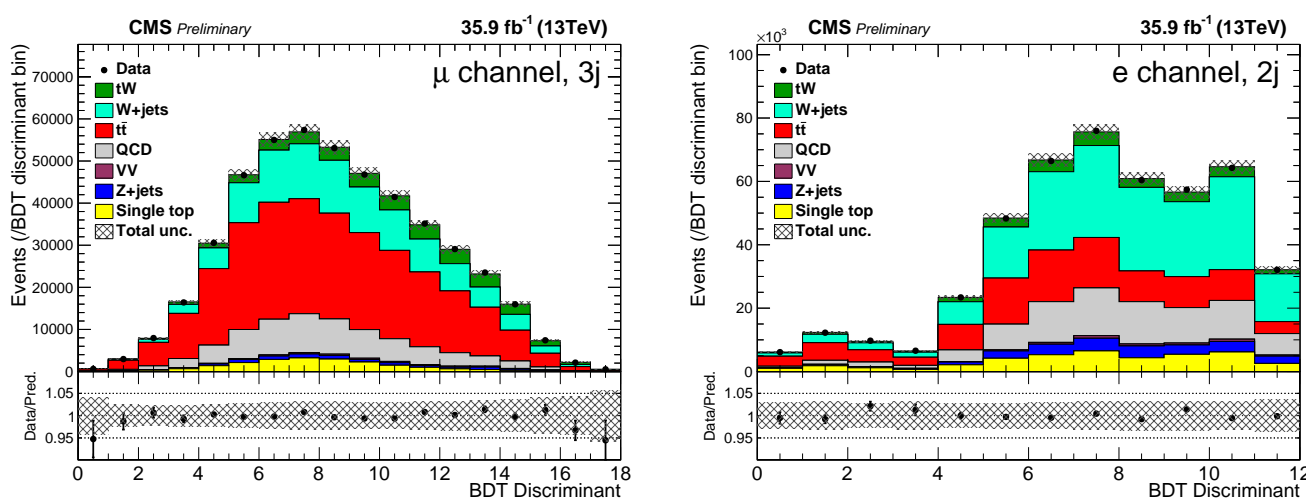

Figure 3: Post fit BDT discriminant in the signal region for the muon (left [1]) and electron (right [1]) channels for the $3 \mathrm{~J} 1 \mathrm{~T}$ and $2 \mathrm{~J} 1 \mathrm{~T}$ regions respectively.

\section{Results}

The mass of the top quark measured using $t$-channel single top quark events for inclusive of the lepton charge in the final state, is given by

$$
m_{\mathrm{t}}=172.13 \pm 0.32(\text { stat }+ \text { prof })_{-0.70}^{+0.69}(\text { syst }) \mathrm{GeV}=172.13_{-0.77}^{+0.76} \mathrm{GeV}
$$

reaching a sub-GeV precision for the first time in such a phase space. The masses of the top quark and antiquark are determined separately by requiring positively and negatively charged leptons in the final state. The mass ratio and difference of the top antiquark to quark is determined to be $0.9952_{-0.0104}^{+0.0079}$ and $0.83_{-1.35}^{+1.79} \mathrm{GeV}$. This systematic uncertainty is dominated by jet energy scale, parton shower scale, and b-quark hadronization model.

The observed tW signal strength is measured as $\mu=1.24 \pm 0.18$, with an expected uncertainty of $17 \%$. This corresponds to an observed and expected excess of signal over the background-only hypothesis exceeding five standard deviations. The measured inclusive production cross section is $89 \pm 4$ (stat) \pm 12 (syst) pb. 


\section{References}

[1] CMS Collaboration. Measurement of tW production in the semileptonic channel in PP collisions at $\sqrt{s}=13 \mathrm{TeV}$. ArXiv e-prints, 2021. arXiv:2109.01706.

[2] CMS Collaboration. Measurement of the top quark mass using events with a single reconstructed top quark in pp collisions at $\sqrt{s}=13 \mathrm{TeV}$. ArXiv e-prints, 2021. arXiv:2108.10407.

[3] M. Czakon, P. Fiedler, and A. Mitov. Total top-quark pair-production cross section at hadron colliders through $O\left(\alpha_{S}\right)$. Phys. Rev. Lett., 110:252004, 2013. arXiv:1303.6254, doi: 10.1103/PhysRevLett. 110.252004 .

[4] H. Ikeda et al. A detailed test of the CsI(Tl) calorimeter for BELLE with photon beams of energy between $20 \mathrm{MeV}$ and $5.4 \mathrm{GeV}$. Nucl. Instrum. Meth. A, 441:401, 2000. doi: 10.1016/S0168-9002(99)00992-4.

[5] A. M. Sirunyan et al. (cms collaboraion), Measurement of the differential cross sections for the associated production of a $\mathrm{W}$ boson and jets in proton-proton collisions at $\sqrt{s}=13 \mathrm{TeV}$. Phys. Rev. D, 96:072005, 2017. arXiv: 1707.05979, doi:10.1103/PhysRevD. 96.072005.

[6] A. M. Sirunyan et al. (cms collaboraion), Measurement of differential cross sections for $\mathrm{Z}$ boson production in association with jets in proton-proton collisions at $\sqrt{s}=13 \mathrm{TeV}$. Eur. Phys. J. C, 78:965, 2018. arXiv: 1804.05252, doi:10.1140/epjc/s10052-018-6373-0.

[7] A. M. Sirunyan et al. (cms collaboraion), Measurement of the single top quark and antiquark production cross sections in the $t$ channel and their ratio in proton-proton collisions at $\sqrt{\mathrm{s}}=$ 13 TeV. Phys. Lett. B, 800:135042, 2020. arXiv: 1812.10514, doi: 10.1016/j . physletb. 2019.135042.

[8] T. Skwarnicki. A study of the radiative CASCADE transitions between the Upsilon-Prime and Upsilon resonances. PhD thesis, Cracow, INP, 1986. URL: http://www-library.desy . de/cgi-bin/showprep.pl?DESY-F31-86-02. 